Document downloaded from:

http://hdl.handle.net/10251/18474

This paper must be cited as:

Ballester Bolinches, A.; Esteban Romero, R. (2002). On a question of Beidleman and Robinson. Communications in Algebra. 12(30). doi:10.1081/AGB-120016008

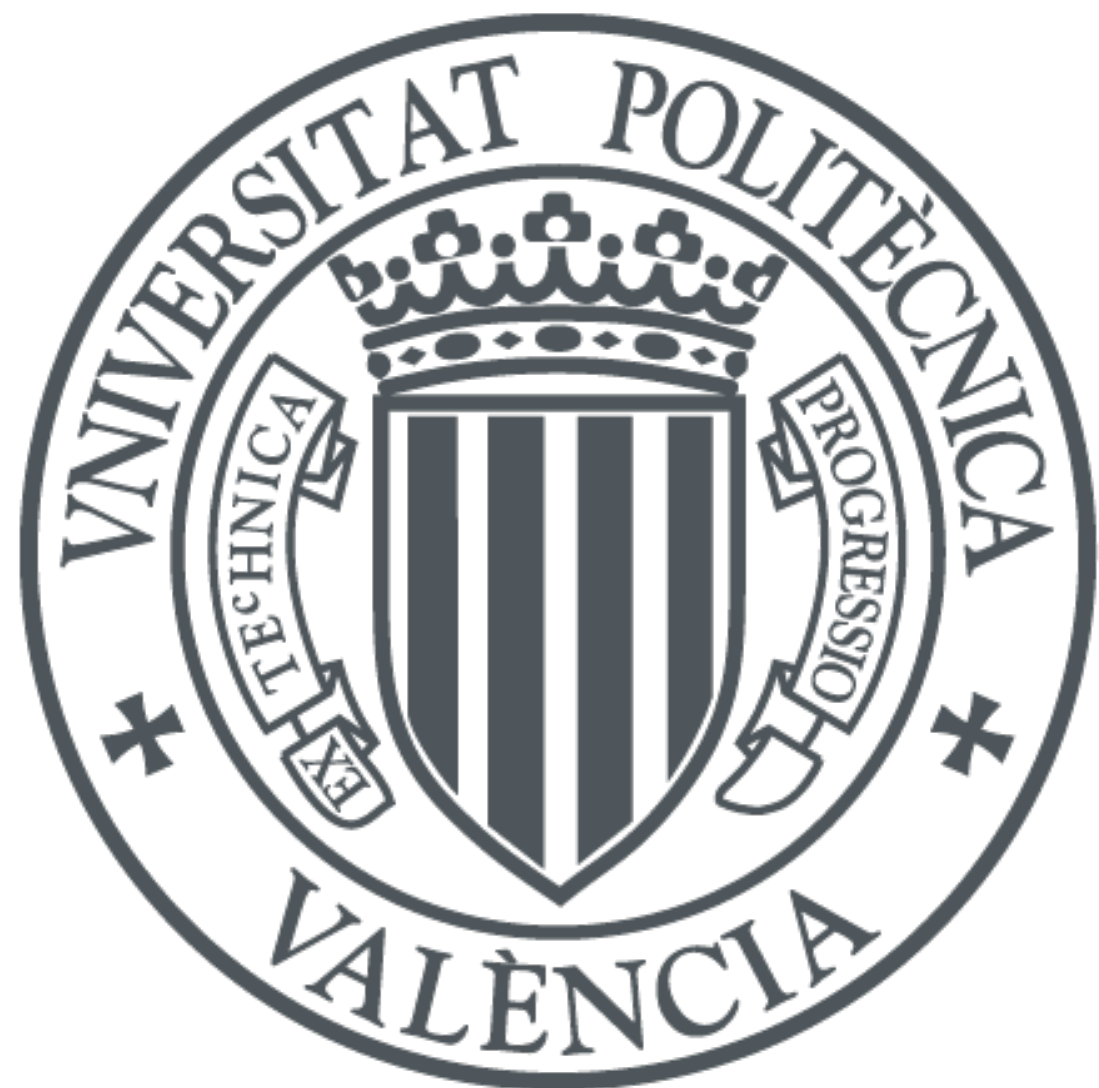

The final publication is available at

http://www.tandfonline.com/10.1081/AGB-120016008

Copyright Taylor \& Francis

Additional Information

[EN] This is an Author's Original Manuscript of an article submitted for consideration in Communications in Algebra 30 (12):5757-5770 (2012) (copyright Taylor \& Francis). Communications in Algebra is available online at http://tandfonline.com/10.1081/AGB120016008 
This is an Author's Original Manuscript of an article submitted for consideration in Communications in Algebra 30(12):5757-5770 (2002) (copyright Taylor \& Francis).

Communications in Algebra is available online at

http://www. tandfonline.com/10.1081/AGB-120016008

DOI 10.1081/AGB-120016008 


\title{
ON A QUESTION OF BEIDLEMAN AND ROBINSON*
}

\author{
A. Ballester-Bolinches \\ Departament d'Àlgebra \\ Universitat de València \\ Dr. Moliner, 50 \\ E-46100 Burjassot (València) \\ Spain \\ email: Adolfo.Ballester@uv.es \\ R. Esteban-Romero \\ Departament de Matemàtica Aplicada \\ Universitat Politècnica de València \\ Camí de Vera, s/n \\ E-46022 València \\ Spain \\ email: resteban@mat.upv.es
}

\begin{abstract}
In (1, Theorem A), Beidleman and Robinson proved that if a group satisfies the permutizer condition, it is soluble, its chief factors have order a prime number or 4 and $G$ induces the full group of automorphisms in the chief factors of order 4. In this paper, we show that the converse of this theorem is false by showing some counterexamples. We also find some sufficient conditions for a group satisfying the converse of that theorem to satisfy the permutizer condition.
\end{abstract}

*Supported by Proyecto PB97-0674-C02-02 and Proyecto PB97-0604 from DGICYT, Ministerio de Educación y Ciencia 


\section{INTRODUCTION}

All groups considered in this paper are finite.

Given a subgroup $H$ of a group $G$, the permutizer $P_{G}(H)$ of $H$ in $G$ is defined as the subgroup generated by all cyclic subgroups of $G$ that permute with $H$. Thus $H \leq P_{G}(H)$ and $H \neq P_{G}(H)$ if and only if $H\langle x\rangle=\langle x\rangle H$ for some $x \in G \backslash H$. A group $G$ such that $H \neq P_{G}(H)$ for every proper subgroup $H$ is said to satisfy the permutizer condition or to be a $\mathbf{P}$-group.

Beidleman and Robinson (1, Theorem A) proved the following result:

Theorem 1. Let $G$ be a finite group satisfying the permutizer condition. Then $G$ is soluble and each chief factor of $G$ has order 4 or a prime. In addition, if $F$ is a chief factor of order 4 , then $G$ induces the full group of automorphisms in $F$, i. e., $G / C_{G}(F) \cong \Sigma_{3}$.

In the same paper, the authors asked whether the converse is true. We show in this paper that the converse is not true and find sufficient conditions for a group satisfying the converse of that theorem to be a $\mathbf{P}$-group.

\section{A COUPLE OF EXAMPLES}

In this section we present a couple of examples to show that the converse of Theorem 1 is false.

Example 1. Let $V$ be an irreducible and faithful $\langle b\rangle$-module over the field of 2 elements, where $\langle b\rangle$ is a cyclic group of order 3 , such that the corresponding semidirect product is isomorphic to $A_{4}$, the alternating group of degree 4 . Let $A_{1}$ and $A_{2}$ be two copies of $V$, and consider $A=A_{1} \times A_{2}$. It is clear that $A$ is a faithful $\langle b\rangle$-module. Denote $B=[A]\langle b\rangle$ the corresponding semidirect product. Then we can choose generators $c, d$ of $A_{1}$ and $e, f$ of $A_{2}$ such that $c^{b}=c d, d^{b}=c, e^{b}=e f$ and $f^{b}=e$. The group $B$ has an automorphism $a$ of order 2 such that $b^{a}=b^{2}, c^{a}=e f, d^{a}=f, e^{a}=c d$ and $f^{a}=d$, so we can consider the semidirect product $G=[B]\langle a\rangle$.

The normal series

$$
1<N=\langle c e f, d e\rangle<A<B<G
$$

is a chief series of $G$. Hence the chief factors of $G$ have order 2, 3 or 4 . On the other hand, $C_{G}(N)=A, C_{G}(A / N)=A, C_{G}(B / A)=B$ and $C_{G}(G / B)=G$. Consequently, $G$ satisfies the converse of Theorem 1.

Let $H=\left[A_{2}\right]\langle b\rangle$. We show that $P_{G}(H)=H$. Suppose there exists an element $x \in G$ of order 3 such that $H\langle x\rangle$ is a subgroup of $G$. Then there 
exists a Sylow 3-subgroup $H_{3}$ of $H$ such that $H_{3}\langle x\rangle$ is a Sylow 3 -subgroup of $H\langle x\rangle$. Since Sylow 3-subgroups of $G$ have order 3, it follows that $H_{3}=\langle x\rangle$ and $x \in H$.

Suppose now that there exists a 2-element $y \in G$ such that $H\langle y\rangle$ is a subgroup of $G$. It is clear that in this case $A_{2}\langle y\rangle$ is a Sylow 2-subgroup of $H\langle y\rangle$. If $y \in A$, then $A_{2}\langle y\rangle$ is an elementary abelian 2-group normalized by $b$. Hence, if $A_{2} \neq A_{2}\langle y\rangle$, there exists an element $z \in A_{2}\langle y\rangle$ such that $A_{2}\langle y\rangle=A_{2} \times\langle z\rangle$ and $b \in C_{G}(\langle z\rangle)$, a contradiction. Therefore $A_{2}\langle y\rangle=A_{2}$ and $y \in H$. Assume that $y=y_{1} a$ for some $y_{1} \in A$. Notice that $y^{2} \in A$ and so $A \cap A_{2}\langle y\rangle=A_{2}\left\langle y^{2}\right\rangle$. Thus $A_{2}\left\langle y^{2}\right\rangle \leq A$ is a normal subgroup of $A_{2}\langle y\rangle$. In particular, $A_{1}=A_{2}^{y}=A_{2}^{a} \leq A_{2}\left\langle y^{2}\right\rangle$, a contradiction.

Finally, assume that there exists an element $g \in G$ such that $H\langle g\rangle$ is a subgroup of $G$. Then $\langle g\rangle=\left\langle g_{1}\right\rangle \times\left\langle g_{2}\right\rangle$, where $\left|\left\langle g_{1}\right\rangle\right|$ is a 2-number and $\left|\left\langle g_{2}\right\rangle\right| \in\{1,3\}$. We can find a Sylow 3-subgroup $H_{3}$ of $H$ such that $H_{3}\left\langle g_{2}\right\rangle$ is a Sylow 3-subgroup of $H\langle g\rangle$. Therefore $H_{3}=\left\langle g_{2}\right\rangle$ and $g_{2} \in H$ because Sylow 3 -subgroups of $G$ have order 3. Hence $H\langle g\rangle=H\left\langle g_{2}\right\rangle$ and so $g_{2} \in H$ by the above case. Consequently $g \in H$ as we want to prove.

Our next example is quite surprising bearing in mind the results of the next section.

Theorem 2. $G=\Sigma_{4} \times \Sigma_{4} \times \Sigma_{4} \times \Sigma_{4}$ is not a $\mathbf{P}$-group.

Proof. It is enough to find a proper subgroup $H$ of $G$ such that $P_{G}(H)=H$. Denote by $G_{i}, 1 \leq i \leq 4$, the factors of $G$ isomorphic to $\Sigma_{4}$ and by $N_{i}=$ $\left\langle a_{i}, b_{i}\right\rangle$ the unique minimal normal subgroup of $G_{i}$. Let $g_{i}$ be an element of order 3 in $G_{i}$ such that $L_{i}=N_{i}\left\langle g_{i}\right\rangle$ is isomorphic to $A_{4}$.

We consider the set $H=H_{1}\langle g\rangle$, where

$$
H_{1}=\left\langle a_{1} a_{3} a_{4}, a_{1}^{g_{1}} a_{3}^{g_{3}} a_{4}^{g_{4}}, a_{2} a_{3} a_{4}^{g_{4}}, a_{2}^{g_{2}} a_{3}^{g_{3}} a_{4}^{g_{4}^{2}}\right\rangle
$$

and $g=g_{1} g_{2} g_{3} g_{4}$. Since $a_{i}^{g_{i}^{2}}=a_{i} a_{i}^{g_{i}}$ for all $i$, it follows that $g \in N_{G}\left(H_{1}\right)$. Hence $H$ is a subgroup of $G$. We prove that $P_{G}(H)=H$.

First of all, $H$ is not permutable with any subgroup of order 3 which is not contained in $H$. Assume not and let $q \in G \backslash H$ be an element of order 3 such that $H\langle q\rangle \leq G$. Then $H_{1}$ is a Sylow 2-subgroup of $H\langle q\rangle$ which is normalized by $q$. Consequently, $a^{q}, b^{q} \in H_{1}$, where $a=a_{1} a_{3} a_{4}$ and $b=a_{2} a_{3} a_{4}^{g_{4}}$. Notice that $H_{1}=\left\langle a, a^{g}\right\rangle \times\left\langle b, b^{g}\right\rangle$. Since the component of $a^{q}$ in $G_{2}$ is trivial and the component of $b^{q}$ in $G_{1}$ is also trivial, it follows that $a^{q} \in\left\langle a, a^{g}\right\rangle$ and $b^{q} \in\left\langle b, b^{g}\right\rangle$. Hence either $a^{q}=a, a^{q}=a^{g}$ or $a^{q}=a a^{g}$, and either $b^{q}=b$, $b^{q}=b^{g}$ or $b^{q}=b b^{g}$. If $q=q_{1} q_{2} q_{3} q_{4}$, with $q_{i} \in G_{i}, 1 \leq i \leq 4$, then one of the following cases holds: 
Case (a) $a_{1}^{q_{1}}=a_{1}, a_{2}^{q_{2}}=a_{2}, a_{3}^{q_{3}}=a_{3}, a_{4}^{q_{4}}=a_{4}, a_{4}^{g_{4} q_{4}}=a_{4}^{g_{4}}$.

Case (b) $a_{1}^{q_{1}}=a_{1}^{g_{1}}, a_{2}^{q_{2}}=a_{2}^{g_{2}}, a_{3}^{q_{3}}=a_{3}^{g_{3}}, a_{4}^{q_{4}}=a_{4}^{g_{4}}, a_{4}^{g_{4} q_{4}}=a_{4} a_{4}^{g_{4}}$.

Case (c) $a_{1}^{q_{1}}=a_{1} a_{1}^{g_{1}}, a_{2}^{q_{2}}=a_{2} a_{2}^{g_{2}}, a_{3}^{q_{3}}=a_{3} a_{3}^{g_{3}}, a_{4}^{q_{4}}=a_{4} a_{4}^{g_{4}}, a_{4}^{g_{4} q_{4}}=a_{4}$.

The case (a) is impossible because the Sylow 3-subgroups of $G_{i}$ act fixedpoint freely on $N_{i}$ for all $i$. Suppose that (b) holds. Then $q_{i} g_{i}^{-1} \in C_{L_{i}}\left(a_{i}\right)=$ $N_{i}$ for all $i$. Therefore $q g^{-1} \in H\langle q\rangle \cap \operatorname{Soc}(G)=H_{1}$ and $q \in H_{1}\langle q\rangle=H$, a contradiction. The case (c) is analogous.

Assume that $H$ is permutable with a subgroup $\langle s\rangle$ contained in $\operatorname{Soc}(G)$. Then, if $s \notin H_{1}$, we have that $H_{1} \times\langle s\rangle$ is a Sylow 2-subgroup of $H\langle s\rangle$ which is normalized by $g$. This means that there exists a subgroup $\langle z\rangle \leq H_{1} \times\langle s\rangle$ such that $g \in C_{G}(\langle z\rangle)$, a contradiction. Hence $s \in H$.

If $H$ is permutable with a subgroup $\langle x\rangle$ of order 2, with $x \in G \backslash V$, where $V=O_{\{2,3\}}(G)$, then $|H\langle x\rangle: H|=2$ and so $H$ must be normalized by $x$. In particular, $x \in N_{G}\left(H_{1}\right)$. Arguing as above, we have that $a^{x} \in\left\{a, a^{g}, a a^{g}\right\}$ and $b^{x} \in\left\{b, b^{g}, b b^{g}\right\}$. Consequently one of the following cases holds if $x=$ $x_{1} x_{2} x_{3} x_{4}$ with $x_{i} \in G_{i}$ :

Case $1 a_{1}^{x_{1}}=a_{1}, a_{2}^{x_{2}}=a_{2}, a_{3}^{x_{3}}=a_{3}, a_{4}^{x_{4}}=a_{4}, a_{4}^{g_{4} x_{4}}=a_{4}^{g_{4}}$.

Case $2 a_{1}^{x_{1}}=a_{1}^{g_{1}}, a_{2}^{x_{2}}=a_{2}^{g_{2}}, a_{3}^{x_{3}}=a_{3}^{g_{3}}, a_{4}^{x_{4}}=a_{4}^{g_{4}}, a_{4}^{g_{4} x_{4}}=a_{4} a_{4}^{g_{4}}$.

Case $3 a_{1}^{x_{1}}=a_{1} a_{1}^{g_{1}}, a_{2}^{x_{2}}=a_{2} a_{2}^{g_{2}}, a_{3}^{x_{3}}=a_{3} a_{3}^{g_{3}}, a_{4}^{x_{4}}=a_{4} a_{4}^{g_{4}}, a_{4}^{g_{4} x_{4}}=a_{4}$.

Suppose that either Case 2 or Case 3 holds. Then the automorphism induced by $x_{4}$ on $N_{4}$ has order 3 , a contradiction. Consequently, Case 1 must hold. In this case, $x_{4}$ centralizes $N_{4}$. Hence $x_{4}=1$. Since $\left(a_{1} a_{3} a_{4}\right)^{g x}=$ $a_{1}^{g_{1} x_{1}} a_{3}^{g_{3} x_{3}} a_{4}^{g_{4}}$ is an element of $H_{1}$, we have that $a_{1}^{g_{1} x_{1}} a_{3}^{g_{3} x_{3}} a_{4}^{g_{4}}=a_{1}^{g_{1}} a_{3}^{g_{3}} a_{4}^{g_{4}}$. Hence $x_{1}$ centralizes $N_{1}$ and $x_{3}$ centralizes $N_{3}$. This implies that $x_{1}=x_{3}=1$. The same argument applied to $\left(a_{2} a_{3} a_{4}^{g_{4}}\right)^{g x}$ shows that $x_{2}=1$. Therefore $x=1$, a contradiction.

Assume now that $H$ is permutable with a cyclic subgroup $\langle x\rangle$ of order 4 which is not contained in $H$. It is clear that $x^{2} \in \operatorname{Soc}(G)$. If $x^{2} \notin H$, then $H \cap\langle x\rangle=1$ and $|H\langle x\rangle: H|=4$. Notice that $\left(x^{2}\right)^{g}$ and $\left(x^{2}\right)^{g^{2}}$ are not in $H$. Therefore $H\langle x\rangle$ is equal to the disjoint union $H\langle x\rangle=H \cup H x^{2} \cup H\left(x^{2}\right)^{g} \cup$ $H\left(x^{2}\right)^{g^{2}}$. This is a contradiction, because $x$ does not belong to this union.

Consequently, $x^{2} \in H \cap \operatorname{Soc}(G)$ and $|H\langle x\rangle: H|=2$. This implies that $x$ normalizes $H$ and so $x$ also normalizes $H_{1}$.

Arguing as in the above paragraph, if $x=x_{1} x_{2} x_{3} x_{4}$, we have that either $a_{4}^{x_{4}}=a_{4}$ and $a_{4}^{g_{4} x_{4}}=a_{4}^{g_{4}}$, or $a_{4}^{x_{4}}=a_{4}^{g_{4}}$ and $a_{4}^{x_{4} g_{4}}=a_{4} a_{4}^{g_{4}}$, or $a_{4}^{x_{4}}=a_{4} a_{4}^{g_{4}}$ and $a_{4}^{g_{4} x_{4}}=a_{4}$. In the first case, $x_{4}$ centralizes $N_{4}$, and in the second and 
third cases, the automorphism induced by $x_{4}$ on $N_{4}$ is of order 3 . The latter possibility gives a contradiction. Hence the first case holds and then $a_{1}^{x_{1}}=a_{1}$, $a_{2}^{x_{2}}=a_{2}$ and $a_{3}^{x_{3}}=a_{3}$. Moreover, $x_{4} \in \operatorname{Soc}(G)$. Arguing as above with the elements $\left(a_{1} a_{3} a_{4}\right)^{g x}$ and $\left(a_{2} a_{3} a_{4}^{g_{4}}\right)^{g x}$, it follows that $x \in \operatorname{Soc}(G)$. This is a contradiction because $\operatorname{Soc}(G)$ has no elements of order 4 .

We would like to mention at this point that what is proved in the above cases is that if $x$ is either an element of order 2 in $G \backslash V$ or $x$ is an element of order 4 , then $\langle x\rangle$ does not permute with $H_{1}$.

Finally, suppose that $\langle x\rangle=\langle x\rangle_{2} \times\langle x\rangle_{3}$ is a subgroup of $G$ such that $H\langle x\rangle$ is a subgroup of $G$. Then $H_{1}\langle x\rangle_{2}$ is a Sylow 2-subgroup of $H\langle x\rangle$. Suppose that $\langle x\rangle_{2}$ is not contained in $H$. By the above remark, $\langle x\rangle_{2}$ is a subgroup of order 2 contained in $\operatorname{Soc}(G)$. Therefore $H\langle x\rangle \leq L_{1} \times L_{2} \times L_{3} \times L_{4}$, and then $g \in N_{G}\left(H_{1}\langle x\rangle_{2}\right)$. This means that there exists an element $y \in H_{1}\langle x\rangle_{2}$ such that $H_{1}\langle x\rangle_{2}=H_{1} \times\langle y\rangle$ and $g \in C_{G}(\langle y\rangle)$, a contradiction. Thus $\langle x\rangle_{2} \leq H$ and $H\langle x\rangle=H\langle x\rangle_{3}$. Bearing in mind the first case, it follows that $\langle x\rangle_{3} \leq H$ and $\langle x\rangle \leq H$.

Consequently, $P_{G}(H)=H$ and the theorem is proved.

\section{QP-GROUPS}

We say that a group $G$ is a $\mathbf{Q P}$-group (or $G$ satisfies the property $\mathbf{Q P}$ ) if $G$ is soluble, each chief factor of $G$ has order 4 or a prime, and if $A / B$ is a chief factor of $G$ of order 4 , then $G$ induces the full group of automorphisms in $A / B$, i. e., $G / C_{G}(A / B) \cong \Sigma_{3}$.

Recall that in any group $G$, there is a unique maximum normal supersolubly embedded subgroup, denoted here by $Z_{\mathfrak{U}}(G)$. It is known that there is a $G$-invariant series in $Z_{\mathfrak{U}}(G)$ with cyclic factors, while $G / Z_{\mathfrak{U}}(G)$ has no nontrivial normal cyclic subgroups. $Z_{\mathfrak{U}}(G)$ is the $\mathfrak{U}$-hypercentre of $G$, where $\mathfrak{U}$ is the formation of all supersoluble groups (see (2, Section IV.6)).

Beidleman and Robinson proved in $(1,3.1)$ the following result:

Lemma 1. A group $G$ is a $\mathbf{P}$-group if and only if $G / Z_{\mathfrak{U}}(G)$ is a $\mathbf{P}$-group.

In order to prove that a $\mathbf{Q P}$-group is a $\mathbf{P}$-group, one often can assume that the $\mathfrak{U}$-hypercentre is trivial. Hence the following result applies.

Lemma 2. Let $G$ be a QP-group such that $Z_{\mathfrak{U}}(G)=1$. Then:

1. $O_{2^{\prime}}(G)=1$ and $G$ is a $\{2,3\}$-group.

2. $G / F(G)$ is isomorphic to a subgroup of a direct product of $\Sigma_{3}$ and $C_{2}$. Consequently, $G / F(G)$ is an extension of a 3-group $V / F(G)$ by a 2group $G / V$, where $V=O_{\{2,3\}}(G)$. 
3. The supersoluble normalizers of $G$ are exactly the normalizers of the Sylow 3-subgroups of $G$.

Proof. 1. Since every chief factor of $G$ of odd order is cyclic, it follows that $O_{2^{\prime}}(G)$ is supersolubly embedded in $G$. Hence $O_{2^{\prime}}(G) \leq Z_{\mathfrak{U}}(G)=1$. Applying $(1,(3.5)), G$ is a $\{2,3\}$-group.

2. follows from the fact that $F(G)$ is the intersection of the centralizers of the chief factors of $G$.

3. Let $D$ be a supersoluble normalizer of $G$ (for properties of normalizers see, for example, (2, Chapter V)). Then there exists a Hall system $\Sigma=\left\{1, G_{2}, G_{3}, G\right\}$ of $G$, where $G_{2}$ is a Sylow 2-subgroup of $G$ and $G_{3}$ is a Sylow 3-subgroup of $G$, such that $D$ is the supersoluble normalizer associated to $\Sigma$. For each prime $p$, we denote $f(p)$ the formation of all abelian groups whose exponent divides $p-1$ and $v(p)=G^{f(p)}$, the $f(p)$-residual of $G$. It is known $((2$, IV.3.4 $))$ that $f$ is an integrated local definition of $\mathfrak{U}$.

Then, according to (2, V.1.1),

$$
D=\bigcap_{p \in\{2,3\}} N_{G}\left(G_{p^{\prime}} \cap v(p)\right)=N_{G}\left(G_{2^{\prime}} \cap v(2)\right) \cap N_{G}\left(G_{3^{\prime}} \cap v(3)\right) .
$$

Since $f(2)=1$, it follows that $v(2)=G$. Moreover, $v(3)$ is contained in $V$ because $G / V$ is an elementary abelian 2-group. On the other hand, $G_{2} \cap V=F(G)$. Hence $G_{3^{\prime}} \cap v(3)=G_{2} \cap v(3) \leq F(G) \cap v(3) \leq$ $G_{2} \cap v(3)$ and $N_{G}\left(G_{3^{\prime}} \cap v(3)\right)=N_{G}(F(G) \cap v(3))=G$. This implies that there exists a Sylow 3 -subgroup $G_{3}$ of $G$ such that $D=N_{G}\left(G_{3}\right)$. The result now follows from the fact that the supersoluble normalizers are conjugate (see (2, V.2.3)).

The following results turn out to be crucial in the proof of our main theorems.

Lemma 3. Let $G$ be a $\mathbf{Q P}$-group contained in a direct product $S_{1} \times \cdots \times S_{r}$ of $r$ copies of $\Sigma_{4}$ and containing the corresponding direct product $A_{1} \times \cdots \times A_{r}$ of $r$ copies of $A_{4}$. Assume that $H$ is a subgroup of $G$ such that $P_{G}(H)=H$ and let $\mathrm{H}_{2}$ and $\mathrm{H}_{3}$ be, respectively, a Sylow 2-subgroup and a Sylow 3-subgroup of $H$. If $N_{i}$ is the minimal normal subgroup of $G$ contained in $A_{i}$, then:

1. If $N_{i} \cap H \neq 1$, then $A_{i} \leq H$.

2. If $N_{i} \cap H=1$, then $H_{2} \leq C_{G}\left(N_{i}\right)$ and $H_{3} \not \leq C_{G}\left(N_{i}\right)$. 
Proof. 1. Suppose that $N_{i} \cap H \neq 1$ and let $1 \neq a_{i} \in N_{i} \cap H$. Since $N_{i}$ is an elementary abelian 2-group of order 4, we can find an element $1 \neq b_{i} \in N_{i}$ such that $N_{i}=\left\langle a_{i}, b_{i}\right\rangle$. Then $H\left\langle b_{i}\right\rangle=H N_{i}$ and so $b_{i} \in P_{G}(H)=H$. Hence $N_{i}$ is contained in $H$. Let now $\left\langle g_{i}\right\rangle$ be a Sylow 3-subgroup of $A_{i}$. Then $A_{i}=N_{i}\left\langle g_{i}\right\rangle$ and so $H A_{i}=H\left\langle g_{i}\right\rangle$. This means that $g_{i} \in P_{G}(H)=H$. Consequently $A_{i}$ is contained in $H$.

2. Suppose that $N_{i} \cap H=1$. We prove that $H_{2}$ centralizes $N_{i}$. Assume that this is not true. We distinguish two cases:

(a) There exists an element $h \in H \backslash C_{G}\left(N_{i}\right)$ such that the component $h_{i}$ of $H$ in $S_{i}$ has order 2 . If $h$ has a component $h_{j}, j \neq i$, of order 3 , then $h^{3}$ is an element of $H \backslash C_{G}\left(N_{i}\right)$ whose component in $S_{i}$ has order 2 . Hence without loss of generality we may assume that $o(h)=2$ or 4 .

Let $a_{i} \in N_{i} \backslash\{1\}$ such that $a_{i}^{h} \neq a_{i}$. Then $h a_{i}$ is an element of $G$ of order 4 (notice that $\left(h a_{i}\right)^{2}=h^{2} a_{i}^{h} a_{i} \neq 1$ ). In this case, $H\left\langle h a_{i}\right\rangle=$ $H N_{i}$ by order considerations. Consequently $h a_{i} \in P_{G}(H)=H$ and so $a_{i} \in N_{i} \cap H=1$, a contradiction.

(b) No element of $H \backslash C_{G}\left(N_{i}\right)$ has its component in $S_{i}$ of order 2. We may assume that there exists $h \in H_{2} \backslash C_{G}\left(N_{i}\right)$. Suppose that $H_{3}$ does not centralize $N_{i}$ and let $g \in H_{3} \backslash C_{G}\left(N_{i}\right)$. We know that $o\left(h_{i}\right)=4$ and so either $h g$ or $h g^{2}$ has its component in $S_{i}$ of order 2. Moreover $h g \in H \backslash C_{G}\left(N_{i}\right)$ and $h g^{2} \in H \backslash C_{G}\left(N_{i}\right)$ because $h_{i} \notin A_{i}$, a contradiction.

Therefore $H_{3} \leq C_{G}\left(N_{i}\right)=S_{1} \times \cdots \times N_{i} \times \cdots \times S_{r}$ and so the projection of $H_{3}$ in $S_{i}$ is 1 . Assume that the projection of $H_{2}$ in $S_{i}$ is a Sylow 2-subgroup of $S_{i}$. Then there exists an element $x \in$ $H \backslash C_{G}\left(N_{i}\right)$ such that $o\left(x_{i}\right)=2$. This is impossible by (2a). Hence the projection of $H_{2}$ in $S_{i}$ is just $\left\langle h_{i}\right\rangle$. In particular $\left\langle h_{i}^{2}\right\rangle$ permutes with $H$. Thus $h_{i}^{2} \in H \cap N_{i}=1$, a contradiction. Consequently $\mathrm{H}_{2}$ centralizes $\mathrm{N}_{i}$.

If $H_{3} \leq C_{G}\left(N_{i}\right)$, then $N_{i} \leq H$, a contradiction. Hence $H_{3}$ cannot centralize $N_{i}$.

Lemma 4. Let $G$ be a $\mathbf{Q P}$-group such that $Z_{\mathfrak{U}}(G)=1$. Assume that $G$ has $r$ chief factors of order 4 in a given chief series of $G$. Suppose that $\operatorname{Soc}(G)=N_{1} \times \cdots \times N_{r}$, where $N_{i}$ is a minimal normal subgroup of $G$ of order 4 for all $i$. Then the order of a Sylow 3-subgroup of $G$ is at most $3^{r}$. 
Moreover, if the order of a Sylow 3-subgroups of $G$ is exactly $3^{r}$, then $G$ is, up to isomorphism, a subgroup of a direct product of $r$ copies of $\Sigma_{4}$ containing the direct product of the corresponding $r$ copies of the alternating group $A_{4}$.

Proof. Denote $T=\operatorname{Soc}(G)=N_{1} \times \cdots \times N_{r}$. The hypothesis on $G$ implies that $G / T$ is supersoluble. Hence, if $D$ is a supersoluble normalizer of $G$, we have that $G=D T$. But $T$ is abelian, therefore $D \cap T$ is a normal subgroup of $G$ contained in $D$. Hence $D \cap T \leq \operatorname{Core}_{G}(D)=Z_{\mathfrak{U}}(G)=1$ and $T$ complements $D$ in $G$.

Set $F=F(G)$. It is clear that $T$ is contained in $F$. Hence $F=T(F \cap D)$. Since $F \cap D \unlhd D$ and $T \leq C_{G}(F \cap D)$, it follows that $F \cap D \unlhd G$ and so $F \cap D \leq \operatorname{Core}_{G}(D)=1$. This implies that $T=F$. Since $F$ is the intersection of the centralizers of all chief factors of $G$, it is clear that $F \leq$ $C_{G}\left(N_{1}\right) \cap \cdots \cap C_{G}\left(N_{r}\right) \leq C_{G}(F)$. The solubility of $G$ implies that $C_{G}(F) \leq F$. Consequently, $F=C_{G}\left(N_{1}\right) \cap \cdots \cap C_{G}\left(N_{r}\right)$ and $G / F$ can be embedded in a direct product of $r$ copies of $\Sigma_{3}$. In particular, if $G_{3}$ is a Sylow 3-subgroup of $G$, we have that $\left|G_{3}\right| \leq 3^{r}$.

In what follows we assume that $\left|G_{3}\right|=3^{r}$ and $D=N_{G}\left(G_{3}\right)$ (Lemma 2). Set $M_{i}=\prod_{j \neq i} N_{j}$. Then $G=N_{i}\left(M_{i} D\right)$ and $N_{i} \cap\left(M_{i} D\right)=1$. Hence $M_{i} D$ is a maximal subgroup of $G$ and $M_{i} D \cap T=M_{i}(D \cap T)=M_{i}$. Denote $T_{i}=\operatorname{Core}_{G}\left(M_{i} T\right), 1 \leq i \leq r$. Assume that $\bigcap_{i=1}^{r} T_{i} \neq 1$. Then there exists a minimal normal subgroup $N$ of $G$ such that $N \leq \bigcap_{i=1}^{r} T_{i}$. But $N \leq T$, therefore $N \leq T \cap T_{i}=M_{i}$ for all $i$, whence $N \leq \bigcap_{i=1}^{r} M_{i}=1$, a contradiction. Thus $\bigcap_{i=1}^{r} T_{i}=1$ and $G / T_{i}$ is isomorphic to $\Sigma_{4}$ for all $i$. As a consequence, $G$ can be embedded in a direct product of $r$ copies of $\Sigma_{4}$.

On the other hand, $G / C_{G}\left(M_{i}\right)=G / \bigcap_{j \neq i} C_{G}\left(N_{j}\right)$ is isomorphic to a subgroup of the direct product of $G / C_{G}\left(N_{j}\right), j \neq i$. Since $G$ is a QP-group, we have that $G / C_{G}\left(N_{j}\right) \cong \Sigma_{3}$ for all $j$. Consequently $\left|G / C_{G}\left(M_{i}\right)\right|_{3} \leq 3^{r-1}$ $\left(n_{3}\right.$ denotes the 3-part of the number $n$ ). Since $\left|G_{3}\right|=3^{r}$, it follows that $\left|C_{G}\left(M_{i}\right)\right|_{3} \geq 3$. In particular, if $B_{i}=G_{3} \cap C_{G}\left(M_{i}\right)$, we have that $B_{i}$ is a nontrivial Sylow 3-subgroup of $C_{G}\left(M_{i}\right)$ and $\left|B_{i}\right| \geq 3$. Moreover, $B_{1} B_{2} \cdots B_{r}$ is a Sylow 3-subgroup of $M_{1} \cdots M_{r}$ and $B_{i} \cap\left(\prod_{j \neq i} B_{j}\right) \leq G_{3} \cap C_{G}\left(M_{i}\right) \cap C_{G}\left(N_{i}\right) \leq$ $G_{3} \cap F=1$. Therefore $\left|B_{i}\right|=3$ for all $i$. It is clear that $B_{i}$ is not contained in $C_{G}\left(N_{i}\right)$. Therefore $N_{i} B_{i}$ is isomorphic to $A_{4}$.

Let $S_{i}=\bigcap_{j \neq i} T_{j}$. We show that if $V=O_{\{2,3\}}(G)$, then $V \cap S_{i}=N_{i} B_{i}$. Since $N_{j} \cap T_{j}=1$ for all $j$, we have that $S_{i}=\bigcap_{j \neq i} T_{j} \leq \bigcap_{j \neq i} C_{G}\left(N_{j}\right)=$ $C_{G}\left(M_{i}\right)$, so that there exists a Sylow 3-subgroup $\left(S_{i}\right)_{3}$ of $S_{i}$ such that $\left(S_{i}\right)_{3} \leq$ $B_{i}$. If $\left(S_{i}\right)_{3}=1$, then $S_{i}$ is a 2-group. Hence a Sylow 3-subgroup of $G / S_{i}$ has order $3^{r}$. This is not possible because $G / S_{i}$ is isomorphic to a direct product of $r-1$ copies of $\Sigma_{4}$. Therefore $\left(S_{i}\right)_{3}=B_{i}$, and hence $N_{i} B_{i} \leq S_{i} \cap V$. Let us prove now that $S_{i} \leq N_{i} D$. We may assume, without loss of generality, 
that $i=1$. We have that $T_{2} \cap \cdots \cap T_{r} \leq M_{2} D \cap M_{3} D \cap \cdots \cap M_{r} D=$ $N_{1} D\left(N_{3} \cdots N_{r} \cap M_{3} D \cap \cdots \cap M_{r} D\right)$. Since $N_{3} \cdots N_{r} \leq T$, it follows that $\left(N_{3} \cdots N_{r}\right) \cap M_{3} D \cap \cdots \cap M_{r} D=\left(N_{3} \cdots N_{r}\right) \cap M_{3} D \cap \cdots \cap M_{r} D \cap T=$ $\left(N_{3} \cdots N_{r}\right) \cap M_{3} \cap \cdots \cap M_{r}=1$. Hence $S_{1} \leq N_{1} D$ and, analogously, $S_{i} \leq N_{i} D$ for all $i$. This implies that $S_{i} \cap V \leq N_{i} D \cap V=N_{i}(D \cap V)=N_{i} G_{3}$ (notice that $\left.D \cap V=G_{3}\right)$. Consequently $S_{i} \cap V \leq N_{i} G_{3} \cap C_{G}\left(M_{i}\right)=N_{i}\left(G_{3} \cap\right.$ $\left.C_{G}\left(M_{i}\right)\right)=N_{i} B_{i}$ and $S_{i} \cap V=N_{i} B_{i}$. Notice that $G_{3}=B_{1} \times \cdots \times B_{r}$. Hence $V=N_{1} B_{1} \cdots N_{r} B_{r}$ by order considerations. Moreover, $N_{i} B_{i} \leq \bigcap_{j \neq i} T_{j}$ and $\left\langle N_{1} B_{1}, \ldots, N_{i-1} B_{i-1}, N_{i+1} B_{i+1}, \ldots, N_{r} B_{r}\right\rangle$ is contained in $T_{i}$. Therefore $N_{i} B_{i} \cap\left\langle N_{1} B_{1}, \ldots, N_{i-1} B_{i-1}, N_{i+1} B_{i+1}, \ldots, N_{r} B_{r}\right\rangle \leq T_{1} \cap \cdots \cap T_{r}=1$ and $V=N_{1} B_{1} \times \cdots \times N_{r} B_{r}$. Consequently $G$ is isomorphic to a subgroup of the direct product of $r$ copies of $\Sigma_{4}$ containing the corresponding direct product of copies of $A_{4}$.

\section{SUFFICIENT CONDITIONS FOR A QP- GROUP TO BE A P-GROUP}

We begin with the following elementary lemma.

Lemma 5. Let $H$ be a subgroup of $G$ and let $N$ be a normal subgroup of $G$ contained in $H$. Then:

1. If $x N \in P_{G / N}(H / N)$, then $x \in P_{G}(H)$.

2. If $P_{G}(H)=H$, then $P_{G / N}(H / N)=H / N$.

Proof. It is clear that 2 is a consequence of 1 . Hence only 1 must be proved. Let $x N \in P_{G / N}(H / N)$. Then $(H / N)\langle x N\rangle=\langle x N\rangle(H / N)$. Let $h x^{a}$ be an element of $H\langle x\rangle$. Then $(h N)(x N)^{a} \in\langle x N\rangle(H / N)$, whence $(h N)(x N)^{a}=$ $(x N)^{b}\left(h^{\prime} N\right)$ for some $h^{\prime} \in H$ and there exists an element $n \in N$ such that $h x^{a}=x^{b} h^{\prime} n$. Since $N \leq H$, it follows that $h x^{a} \in\langle x\rangle H$. Consequently $H\langle x\rangle \subseteq\langle x\rangle H$. The other inclusion is analogous.

Our aim in this section is to find sufficient conditions for a QP-group to be a $\mathbf{P}$-group in terms of the number of the chief factors of order 4 .

Theorem 3. Let $G$ be a QP-group with just one chief factor of order 4 in a given chief series of $G$. Then $G$ is a $\mathbf{P}$-group.

Proof. Let $D \neq G$ be a supersoluble normalizer of $G$. Then, by (2, V.3.8), $D$ can be joined with a chain of subgroups:

$$
D=D_{0}<\cdot D_{1}<\cdots<\cdot D_{r}=G
$$


such that $D_{i-1}$ is a maximal subgroup of $D_{i}, D_{i} / \operatorname{Core}_{D_{i}}\left(D_{i-1}\right)$ is not supersoluble and $D_{i}=D_{i-1} F\left(D_{i}\right)$ for all $i$. Moreover, $D$ covers all the chief factors of $G$ of prime order and avoids the chief factors of $G$ of order 4 . Since $G$ has only one chief factor of order 4 , we conclude that $D$ is a maximal subgroup of $G$. Since $G / \operatorname{Core}_{G}(D)$ is not supersoluble, it follows that $G / \operatorname{Core}_{G}(D) \cong \Sigma_{4}$. By $(2, \mathrm{~V} .2 .4), Z_{\mathfrak{U}}(G)=\operatorname{Core}_{G}(D)$. Hence $G / Z_{\mathfrak{U}}(G)$ is a $\mathbf{P}$-group. By Lemma $1, G$ is a $\mathbf{P}$-group. If $D=G$, then $G$ is supersoluble and so $G$ is a $\mathbf{P}$-group.

To find sufficient conditions for a QP-group $G$ with more than one chief factor in a given chief series to be $\mathbf{P}$-group seems to be quite difficult. However we have obtained some interesting results when $\operatorname{Soc}(G)$ is a direct product of minimal normal subgroups of $G$ of order 4 .

Theorem 4. Assume that $G$ is a $\mathbf{Q P}$-group contained in a direct product $S_{1} \times S_{2}$ of two copies of $\Sigma_{4}$ and containing the direct product $A_{1} \times A_{2}$ of the copies of $A_{4}$. Then $G$ is a $\mathbf{P}$-group.

Proof. Denote by $N_{i}, i=1,2$, the minimal normal subgroups of $G$ contained in $A_{1} \times A_{2}$.

Assume that $G$ is not a $\mathbf{P}$-group and let $H$ be a proper subgroup of $G$ such that $H=P_{G}(H)$. If $H \cap N_{i} \neq 1$ for some $i$, then $A_{i} \leq H$ by Lemma 3 . It is clear that $G / A_{i}$ is isomorphic to either $\Sigma_{4}$ or $\Sigma_{4} \times C_{2}$, which are both $\mathbf{P}$-groups. By Lemma 5, it follows that $H=G$, a contradiction. Hence $H \cap N_{1}=1=H \cap N_{2}$. Bearing in mind the notation of Lemma 3, we have that $H_{2} \leq C_{G}\left(N_{i}\right)$ and $H_{3} \neq C_{G}\left(N_{i}\right)$ for $i=1,2$.

Suppose first that $H_{3}=\left\langle g_{1} g_{2}\right\rangle$, where $g_{1} \in A_{1}, g_{2} \in A_{2}$ are elements of order 3. Assume that $H \cap \operatorname{Soc}(G)=1$, then $H_{2}=1$ because $H_{2} \leq$ $C_{G}(\operatorname{Soc}(G))=\operatorname{Soc}(G)$. Hence $H=H_{3}$. Let $h_{i} \in S_{i}$ be an element of order 2 such that $g_{i}^{h_{i}}=g_{i}^{-1}, i=1,2$. Then $h_{1} h_{2} \in G$ because $G$ is a QP-group. It is clear that $h_{1} h_{2} \in N_{G}(H)=H$ and so $H_{2} \neq 1$, a contradiction. Therefore $H \cap \operatorname{Soc}(G) \neq 1$.

Since $H_{2} \leq \operatorname{Soc}(G)$, it follows that $H \cap \operatorname{Soc}(G)=H_{2}$. Hence $H_{2} \unlhd H$ and $H_{2}$ is an $H_{3}$-module over the field of 2 elements. Let $a_{1} a_{2} \in H_{2}$, with $1 \neq a_{i} \in N_{i}, i=1,2$. Then $a_{1}^{g_{1}} a_{2}^{g_{2}} \in H_{2}$ and $a_{1} a_{2} \neq a_{1}^{g_{1}} a_{2}^{g_{2}}$. Consequently the subgroup $T=\left\langle a_{1} a_{2}, a_{1}^{g_{1}} a_{2}^{g_{2}}\right\rangle$ has order 4 . Suppose that $T<H_{2}$. Since $H_{2}$ is a completely reducible $H_{3}$-module, there exists a normal subgroup $T_{0}$ of $H$ such that $H_{2}=T \times T_{0}$. Let $c_{1} c_{2}$ be an element belonging to $T_{0}$, where $1 \neq c_{i} \in N_{i}, i=1,2$. Then $c_{1}^{g_{1}} c_{2}^{g_{2}} \in T_{0}$. This implies that $H_{2}$ has order 16 and so $\operatorname{Soc}(G) \leq H$, a contradiction. Therefore $H_{2}=\left\langle a_{1} a_{2}, a_{1}^{g_{1}} a_{2}^{g_{2}}\right\rangle$. Let $h_{1} h_{2}$ be an element of $S_{1} \times S_{2}$ such that $o\left(h_{i}\right)=2, g_{i}^{h_{i}}=g_{i}^{-1}$ and $a_{i}^{h_{i}}=a_{i}^{g_{i}}$ 
for $i=1,2$. Since $G$ is a QP-group, it follows that $h_{1} h_{2} \in G$. It is clear that $h_{1} h_{2} \in N_{G}(H)=H$. This is a contradiction.

Assume that $H_{3}=\left\langle g_{1}, g_{2}\right\rangle$, with $g_{i} \in A_{i}, i=1,2$, of order 3. Let $a_{i} a_{2}$ be an element of $H_{2}=\operatorname{Soc}(G) \cap H$. Then $a_{1}^{g_{1}} a_{2} \in H$ and so $a_{1} a_{1}^{g_{1}} \in H \cap N_{1}=1$. Therefore $a_{1}^{g_{1}}=a_{1}$ and then $a_{1}=1$. Analogously $a_{2}=1$. Hence $H_{2}=1$. Let $h_{1} h_{2} \in G$ be an element of order 2 such that $g_{i}^{h_{i}}=g_{i}^{-1}, i=1,2$. Then $h_{1} h_{2} \in N_{G}(H)=H$. This contradicts the fact $H_{2}=1$.

Consequently $G$ is a $\mathbf{P}$-group.

Theorem 5. Let $G$ be a subgroup of the direct product $\Sigma_{4} \times \Sigma_{4} \times \Sigma_{4}$ of three copies of the symmetric group of degree 4. Assume that $G$ contains $A_{4} \times A_{4} \times A_{4}$, the corresponding direct product of the alternating groups. If $G$ is a QP-group and $G$ contains an element of order 2 inverting all the elements of a Sylow 3-subgroup of $G$, then $G$ is a $\mathbf{P}$-group.

Proof. Denote by $G_{i}, 1 \leq i \leq 3$, the factors of the direct product $\Sigma_{4} \times \Sigma_{4} \times \Sigma_{4}$. Let $N_{i}, 1 \leq i \leq 3$, be the Klein four-group in $G_{i}$. Then $N_{i}$ is a minimal normal subgroup of $G$ for all $i$.

Suppose there exists a proper subgroup $H$ of $G$ such that $P_{G}(H)=H$. If $H \cap N_{i} \neq 1$, then $A_{i}$, the alternating group in $G_{i}$, is contained in $H$. Moreover, $G / A_{i}$ is isomorphic to one of the groups of Theorem 4 or to a direct product of one of these groups with $C_{2}$. Then $G / A_{i}$ is a $\mathbf{P}$-group, a contradiction. Therefore $H \cap N_{1}=H \cap N_{2}=H \cap N_{3}=1$ and, by Lemma 3, every Sylow 2-subgroup $\mathrm{H}_{2}$ of $H$ centralizes $N_{i}$ for all $i$ and none Sylow 3-subgroup $H_{3}$ of $H$ centralizes $N_{i}$ for all $i$. Consequently every Sylow 2-subgroup $\mathrm{H}_{2}$ of $H$ is contained in $C_{G}\left(N_{1} \times N_{2} \times N_{3}\right)=N_{1} \times N_{2} \times N_{3}=\operatorname{Soc}(G)$ and $H_{2}=H \cap \operatorname{Soc}(G)$ is the unique Sylow 2-subgroup of $H$.

Suppose that $H_{3}$ is of order 3 . Then, since $H_{3} \not \leq C_{G}\left(N_{i}\right)$ for all $i$, it follows that $H_{3}=\left\langle g_{1} g_{2} g_{3}\right\rangle$, where $g_{i} \in G_{i}$ is an element of order 3 for all $i$. If $H_{3}=H$, then we consider an element $h_{i} \in G_{i}$ of order 2 such that $g_{i}^{h_{i}}=g_{i}^{-1}$ for all $i$ and $h_{1} h_{2} h_{3} \in G$. Such an element exists because $G$ has an element of order 2 inverting all the elements of a Sylow 3-subgroup of $G$ and $G$ contains the direct product of the alternating groups. Then $h_{1} h_{2} h_{3} \in N_{G}(H)=H$, a contradiction. Consequently $H \cap \operatorname{Soc}(G) \neq 1$. Let $1 \neq a_{1} a_{2} a_{3} \in H \cap \operatorname{Soc}(G)$, with $a_{i} \in N_{i}, i \in\{1,2,3\}$. Notice that $a_{1}^{g_{1}} a_{2}^{g_{2}} a_{3}^{g_{3}} \in H \cap \operatorname{Soc}(G)$. This implies that the rank of $H \cap \operatorname{Soc}(G)$ is a multiple of 2 .

Assume that $H \cap \operatorname{Soc}(G)=\left\langle a_{1} a_{2} a_{3}, a_{1}^{g_{1}} a_{2}^{g_{2}} a_{3}^{g_{3}}\right\rangle$. Let $h_{i} \in G_{i}$ be an element of order 2 such that $g_{i}^{h_{i}}=g_{i}^{-1}$ and $a_{i}^{h_{i}}=a_{i}^{g_{i}}$ for $i \in\{1,2,3\}$. Then $h_{1} h_{2} h_{3} \in G$ and $h_{1} h_{2} h_{3} \in N_{G}\left(H_{2}\right) \cap N_{G}\left(H_{3}\right) \leq N_{G}(H)=H$. In particular, $h_{1} h_{2} h_{3} \in$ $H_{2} \leq C_{G}\left(N_{i}\right)$ for all $i$, a contradiction.

Suppose now that $H \cap \operatorname{Soc}(G)$ has rank 4. Then

$$
H \cap \operatorname{Soc}(G)=\left\langle a_{1} a_{2} a_{3}, a_{1}^{g_{1}} a_{2}^{g_{2}} a_{3}^{g_{3}}, c_{1} c_{2} c_{3}, c_{1}^{g_{1}} c_{2}^{g_{2}} c_{3}^{g_{3}}\right\rangle .
$$


Operating with the generators of $H \cap \operatorname{Soc}(G)$, we can conclude that $H \cap$ $\operatorname{Soc}(G)=\left\langle b_{1} b_{3}, b_{1}^{g_{1}} b_{3}^{g_{3}}, d_{2} d_{3}, d_{2}^{g_{2}} d_{3}^{g_{3}}\right\rangle$ and, by taking a suitable conjugate of $d_{2} d_{3}$, we can also assume that either $d_{3}=b_{3}$ or $b_{3}=1$. Suppose that $d_{3}=b_{3}$ (the case $b_{3}=1$ is similar). Then we can take $h_{1} \in G_{i}$ of order 2 such that $g_{1}^{h_{1}}=g_{1}^{-1}$ and $b_{1}^{h_{1}}=b_{1}^{g_{1}}$, an element $h_{2} \in G_{2}$ of order 2 such that $g_{2}^{h_{2}}=g_{2}^{-1}$ and $d_{2}^{h_{2}}=d_{2}^{g_{2}}$, and an element $h_{3} \in G_{3}$ of order 2 such that $g_{3}^{h_{3}}=g_{3}^{-1}$ and $b_{3}^{h_{3}}=b_{3}^{g_{3}}$. Then the element $h_{1} h_{2} h_{3} \in G$ and it normalizes $H$, a contradiction.

If $H \cap \operatorname{Soc}(G)$ has $\operatorname{rank} 6$, then $\operatorname{Soc}(G) \leq H$, a contradiction.

Assume that $H_{3}$ is of order 9. We can suppose, by reordering the suffices, that either $H_{3}=\left\langle g_{1}, g_{2} g_{3}\right\rangle$ or $H_{3}=\left\langle g_{1} g_{3}, g_{2} g_{3}\right\rangle$.

Suppose that $H_{3}=\left\langle g_{1}, g_{2} g_{3}\right\rangle$. If $1 \neq a_{1} a_{2} a_{3} \in H \cap \operatorname{Soc}(G)$, with $a_{i} \in N_{i}$, $i \in\{1,2,3\}$, we have that $\left(a_{1} a_{2} a_{3}\right)^{g_{1}}=a_{1}^{g_{1}} a_{2} a_{3} \in H$, so their product $a_{1} a_{1}^{g_{1}} \in$ $H \cap N_{1}=1$. This implies that $a_{1}=1$. If $H \cap \operatorname{Soc}(G)$ has rank 4, it follows that $N_{i} \leq H$ for $i \in\{2,3\}$, a contradiction. Hence $H \cap \operatorname{Soc}(G)$ has rank 2 . Then $H \cap \operatorname{Soc}(G)=\left\langle a_{2} a_{3}, a_{2}^{g_{2}} a_{3}^{g_{3}}\right\rangle$. We consider an element $h_{1} \in G_{1}$ of order 2 such that $g_{1}^{h_{1}}=g_{1}^{-1}$, an element $h_{2} \in G_{2}$ of order 2 such that $g_{2}^{h_{2}}=g_{2}^{-1}$ and $a_{2}^{h_{2}}=a_{2}^{g_{2}}$, an an element $h_{3} \in G_{3}$ of order 2 such that $g_{3}^{h_{3}}=g_{3}^{-1}$ and $a_{3}^{h_{3}}=a_{3}^{g_{3}}$. Then $h_{1} h_{2} h_{3} \in G$ and $h_{1} h_{2} h_{3} \in N_{G}(H)=H$, a contradiction.

Suppose that $H_{3}=\left\langle g_{1} g_{3}, g_{2} g_{3}\right\rangle$. If $1 \neq a_{1} a_{2} a_{3} \in H \cap \operatorname{Soc}(G)$, with $a_{i} \in N_{i}, i \in\{1,2,3\}$, we have that $\left(a_{1} a_{2} a_{3}\right)^{g_{1} g_{3}}=a_{1}^{g_{1}} a_{2} a_{3}^{g_{3}} \in H$. Thus $\left(a_{1} a_{2} a_{3}\right)\left(a_{1}^{g_{1}} a_{2} a_{3}^{g_{3}}\right)=a_{1} a_{1}^{g_{1}} a_{3} a_{3}^{g_{3}} \in H$. Then $a_{1} a_{3} \in H$. Therefore $\left(a_{1} a_{3}\right)^{g_{2} g_{3}}=$ $a_{1} a_{3}^{g_{3}} \in H$. This implies that $a_{3} a_{3}^{g_{3}} \in H \cap N_{3}=1$, whence $a_{3}=1$ and $a_{1} \in H \cap N_{1}=1$, a contradiction.

If $H_{3}=\left\langle g_{1}, g_{2}, g_{3}\right\rangle$ and $1 \neq a_{1} a_{2} a_{3} \in H \cap \operatorname{Soc}(G)$, with $a_{i} \in N_{i}, 1 \leq i \leq 3$, then $\left(a_{1} a_{2} a_{3}\right)^{g_{1}}=a_{1}^{g_{1}} a_{2} a_{3} \in H$. Hence $a_{1} a_{1}^{g_{1}} \in H \cap N_{1}=1$. This implies $a_{1}=1$. Analogously $a_{2}=1=a_{3}$. This is a contradiction.

Therefore $G$ is a $\mathbf{P}$-group.

If $G$ is a QP-group such that $A_{4} \times A_{4} \times A_{4} \leq G \leq \Sigma_{4} \times \Sigma_{4} \times \Sigma_{4}$, then $G$ is not a $\mathbf{P}$-group in general, as the next example shows.

Example 2. The direct product $\Sigma_{4} \times \Sigma_{4} \times \Sigma_{4}$ can be considered as a subgroup of $\Sigma_{12}$ and, so viewed, we consider the group $G$ of all even permutations of $\Sigma_{4} \times \Sigma_{4} \times \Sigma_{4}$. Let $a=(1,2)(3,4)(5,6)(7,8)(9,10)(11,12)$ and $g=(1,2,3)(5,6,7)(9,10,11)$. Then $H=\langle a, g\rangle$ is a subgroup of $G$ isomorphic to $A_{4}$. With similar arguments to those used in the proof of Theorem 2 we have that $P_{G}(H)=H$. Therefore $G$ is not a $\mathbf{P}$-group.

Combining Lemma 4 and Theorems 4 and 5 we have:

Theorem 6. Let $G$ be a $\mathbf{Q P}$-group such that $Z_{\mathfrak{U}}(G)=1$. Suppose that $G$ has a chief series with exactly $r$ chief factors of order 4 and $\operatorname{Soc}(G)=$ $N_{1} \times \cdots \times N_{r}$, where $N_{i}$ is a minimal normal subgroup of $G$ of order 4 . If 
either $r=2$ and Sylow 3-subgroups of $G$ have order 9 or $r=3$, Sylow 3subgroups of $G$ have order $3^{3}$ and there exists an element of $G$ of order 2 inverting the elements of a Sylow 3-subgroup of $G$, then $G$ is a $\mathbf{P}$-group.

Theorem 7. Suppose that $G$ is a subgroup of $\Sigma_{4} \times \Sigma_{4}$ containing the product $N_{1} \times N_{2}$ of both minimal normal subgroups of $\Sigma_{4} \times \Sigma_{4}$, and that $G$ is a $\mathbf{P}$-group with $Z_{\mathfrak{U}}(G)=1$. Then $|G|_{3}=9$.

Proof. Let $S_{1}, S_{2}$ be the copies of $\Sigma_{4}$ in $\Sigma_{4} \times \Sigma_{4}$ and $A_{1}, A_{2}$ be the corresponding alternating subgroups.

If $|G|_{3}=1$, then $G$ is a 2-group, whence $Z_{\mathfrak{U}}(G)=G$, a contradiction.

If $|G|_{3}=3$, the projections of a Sylow 3-subgroup $G_{3}$ of $G$ in $S_{i}$ are nontrivial (otherwise $Z_{\mathfrak{U}}(G) \neq 1$ ). Therefore there exist $g_{i} \in S_{i}, i=1,2$, such that $g_{1} g_{2} \in G$. There exist $h_{i} \in S_{i} \backslash A_{i}, i=1,2$, such that $g_{i}^{h_{i}}=g_{i}^{-1}$ and $h_{1} h_{2} \in G$. If $h_{1} \in G$, then $\left(g_{1} g_{2}\right)^{h_{1}}=g_{1}^{-1} g_{2} \in G$, whence $g_{1} \in G$ and $g_{2} \in G$, a contradiction. Hence $h_{1} \notin G$ and, analogously, $h_{2} \notin G$. There exists $a_{i} \in N_{i} \backslash\{1\}$ such that $a_{i}^{h_{i}}=a_{i}^{g_{i}}, i=1,2$. We construct $H=\left\langle a_{1} a_{2}^{g_{2}}, a_{1}^{g_{1}} a_{2}^{g_{2}^{2}}, g_{1} g_{2}\right\rangle$. By arguing like in Example 1, we obtain that $H$ is a proper subgroup of $G$ such that $H=P_{G}(H)$, a contradiction.

Theorem 8. Suppose that $G$ is a subgroup of $\Sigma_{4} \times \Sigma_{4} \times \Sigma_{4}$ containing the direct product of the three minimal normal subgroups of $\Sigma_{4} \times \Sigma_{4} \times \Sigma_{4}$ and that $G$ is a $\mathbf{P}$-group with $Z_{\mathfrak{U}}(G)=1$. Then $|G|_{3}=27$.

Proof. Let $S_{i}, i \in\{1,2,3\}$, be the copies of $\Sigma_{4}$ in $\Sigma_{4} \times \Sigma_{4} \times \Sigma_{4}$ and let $A_{i}$ be the copies of the corresponding alternating subgroups.

If $|G|_{3}=1$, then $G$ is a 2-group, a contradiction with $Z_{\mathfrak{U}}(G)=1$.

If $|G|_{3}=3$, then the projections of a Sylow 3-subgroup $G_{3}$ of $G$ in $S_{i}$ are nontrivial (otherwise, $Z_{\mathfrak{U}}(G) \neq 1$ ). Hence there exist $g_{i} \in S_{i}$ of order 3 , $i \in\{1,2,3\}$, such that $g_{1} g_{2} g_{3} \in G$. There exist $h_{i} \in S_{i} \backslash A_{i}$ of order 2 such that $g_{i}^{h_{i}}=g_{i}^{-1}, i \in\{1,2,3\}$, and $h_{1} h_{2} h_{3} \in G$. If $h_{1} \in G$, then $\left(g_{1} g_{2} g_{3}\right)^{h_{1}}=$ $g_{1}^{-1} g_{2} g_{3} \in G$, whence $g_{1} \in G$, a contradiction. Thus $h_{1} \notin G$ and, analogously, $h_{2} \notin G, h_{3} \notin G$. Consequently $G=\left[N_{1} \times N_{2} \times N_{3}\right]\left\langle g_{1} g_{2} g_{3}, h_{1} h_{2} h_{3}\right\rangle$. Let $a_{i} \in N_{i} \backslash\{1\}$ such that $a_{i}^{h_{i}}=a_{i}^{g_{i}}$ for $i \in\{1,2,3\}$. The subgroup

$$
H=\left\langle a_{1} a_{2} a_{3}^{g_{3}}, a_{1}^{g_{1}} a_{2}^{g_{2}} a_{3}^{g_{3}^{2}}, g_{1} g_{2} g_{3}\right\rangle
$$

is a proper subgroup of $G$ such that $P_{G}(H)=H$, as we can prove like in Example 1, a contradiction.

If $|G|_{3}=9$, then the projections of a Sylow 3-subgroup $G_{3}$ of $G$ in $S_{i}$ are again nontrivial (otherwise, $Z_{\mathfrak{U}}(G) \neq 1$ ). By reordering the suffices, we can suppose that either $G_{3}=\left\langle g_{1} g_{2}, g_{3}\right\rangle$ or $G_{3}=\left\langle g_{1} g_{2}, g_{1} g_{3}\right\rangle$. 
Suppose that $G_{3}=\left\langle g_{1} g_{2}, g_{3}\right\rangle$. There exist elements $h_{i} \in S_{i} \backslash A_{i}, i \in$ $\{1,2,3\}$, of order 2 , such that $g_{i}^{h_{i}}=g_{i}^{-1}$ and $h_{1} h_{2} h_{3} \in G$. If $h_{1} \in G$, then $\left(g_{1} g_{2}\right)^{h_{1}}=g_{1}^{-1} g_{2}$, whence $g_{2} \in G$, a contradiction. Consequently $h_{1} \notin G$ and, analogously, $h_{2} \notin G$. Let $a_{i} \in N_{i} \backslash\{1\}$ such that $a_{i}^{h_{i}}=a_{i}^{g_{i}}, i \in\{1,2,3\}$. If $h_{3} \in G$, then we consider the subgroup

$$
H=\left\langle a_{1} a_{2}^{g_{2}}, a_{1}^{g_{1}} a_{2}^{g_{2}^{2}}, a_{3}, a_{3}^{g_{3}}, g_{1} g_{2}, g_{3}, h_{3}\right\rangle,
$$

and if $h_{3} \notin G$, we take the subgroup

$$
H=\left\langle a_{1} a_{2}^{g_{2}}, a_{1}^{g_{1}} a_{2}^{g_{2}^{2}}, a_{3}, a_{3}^{g_{3}}, g_{1} g_{2}, g_{3}\right\rangle
$$

Like in Example 1 we have that $H$ is a proper subgroup of $G$ such that $P_{G}(H)=H$.

Suppose that $G_{3}=\left\langle g_{1} g_{2}, g_{1} g_{3}\right\rangle$. There exist elements $h_{i} \in S_{i} \backslash A_{i}, i \in$ $\{1,2,3\}$, of order 2, such that $g_{i}^{h_{i}}=g_{i}^{-1}$ and $h_{1} h_{2} h_{3} \in G$. If $h_{1} \in G$, then $\left(g_{1} g_{2}\right)^{h_{1}}=g_{1}^{-1} g_{2} \in G$, whence $g_{1} \in G$, a contradiction. Consequently, $h_{1} \notin G$. Analogously, $h_{2} \notin G$ and $h_{3} \notin G$. Consider an element $a_{i} \in S_{i} \backslash A_{i}$ such that $a_{i}^{h_{i}}=a_{i}^{g_{i}}$ for $1 \leq i \leq 3$. Notice that $\left(g_{1} g_{2}\right)^{2}\left(g_{1} g_{3}\right)^{2}=g_{1} g_{2}^{2} g_{3}^{2}$. The subgroup $H=\left\langle a_{1} a_{2} a_{3}, a_{1}^{g_{1}} a_{2}^{g_{2}^{2}} a_{3}^{g_{3}^{2}}, g_{1} g_{2}^{2} g_{3}^{2}\right\rangle$ is a proper subgroup of $G$ such that $P_{G}(H)=H$.

\section{REFERENCES}

[1] Beidleman, James C.; Robinson, Derek J. S. On finite groups satisfying the permutizer condition. J. Algebra 1997, 191, 686-703.

[2] Doerk, Klaus; Hawkes, Trevor. Finite Soluble Groups; Number 4 in De Gruyter Expositions in Mathematics; Walter de Gruyter: Berlin, New York, 1992.

[3] The GAP Group, Aachen, St Andrews. GAP Groups, Algorithms, and Programming, Version 4.1, 1999. (http://www-gap.dcs.st-and.ac.uk/ gap). 